EPJ Web of Conferences 41, 02021 (2013)

DOI: $10.1051 /$ epjconf/20134102021

(C) Owned by the authors, published by EDP Sciences, 2013

\title{
Fragmentation Control of a Polyatomic Molecule by fully determined Laser-Fields
}

\author{
X. Xie ${ }^{1}$, S. Roither ${ }^{1}$, M. Schöffler ${ }^{1}$, D. Kartashov ${ }^{1}$, H. Xu ${ }^{1,6}$, L. Zhang ${ }^{1}$, T. Rathje ${ }^{2}$, G. G. Paulus ${ }^{2}$, K. \\ Doblhoff-Dier $^{3}$, S. Gräfe ${ }^{3}$, S. Bubin ${ }^{4}$, M. Atkinson ${ }^{4}$, K. Varga ${ }^{4}$, K. Yamanouchi ${ }^{5}$, A. Baltuška ${ }^{1}$, and \\ M. Kitzler ${ }^{1, a}$ \\ 1 Photonics Institute, Vienna University of Technology, A-1040 Vienna, Austria \\ 2 Institute of Optics and Quantum Electronics, D-07743 Jena, Germany \\ 3 Institute of Theoretical Physics, Vienna University of Technology, A-1040 Vienna, Austria \\ 4 Department of Physics and Astronomy, Vanderbilt University, Nashville, Tennessee 37235, USA \\ 5 Department of Chemistry, School of Science, The University of Tokyo, Tokyo 113-0033, Japan \\ 6 State Key Laboratory on Integrated Optoelectronics, Changchun 130012, China
}

\begin{abstract}
Strong-field control of acetylene fragmentation by fully determined fewcycle laser pulses is demonstrated. The control mechanism is shown to be based on electron recollision and inelastic ionization from inner-valence molecular orbitals.
\end{abstract}

Chemistry is usually perceived as breaking and making molecular bonds. These processes that typically occur on the timescale of tens of femtoseconds (fs) to nanoseconds are preceded and ultimately governed by the much faster intra-molecular motion of electrons that proceeds on the sub-femtosecond timescale $[1,2]$. This timescale matches the light oscillations of laser pulses carried at frequencies in the visible and near-infrared. As the electric field of strong laser pulses is capable of exerting forces onto the electrons that are comparable to those of the binding forces, it becomes possible to drive the intra-molecular electron density by light. Deterministic steering, however, requires fully controlled laser electric fields, for example few-cycle pulses with a locked carrier-envelope (CE) offset frequency. Using such pulses it has been shown that the localization of an electron during dissociation of $\mathrm{D}_{2}^{+}[3,4]$ and $\mathrm{CO}^{+}$[5], as well as the directionality of multiple dissociative channels of $\mathrm{CO}$ [6] can be controlled.

In this submission we extend this scheme from simple diatomics studied so far to chemically more relevant polyatomic molecules. Particularly interesting for applications are hydrocarbon molecules. It has been shown previously that dissociation of these molecules is accompanied by extraordinarily rich internal electronic dynamics $[7,8]$ that are still far from being understood.

We study the fragmentation of acetylene, $\mathrm{C}_{2} \mathrm{H}_{2}$, subject to fully determined few-cycle laser electric fields. In our experiments we generate $4.5 \mathrm{fs}$ laser pulses by spectral broadening of $\approx 25 \mathrm{fs}$ laser pulses from a Titanium-Sapphire laser amplifier system in a $1 \mathrm{~m}$ long hollow-core glass capillary filled with argon and subsequent recompression by several bounces from chirped mirrors. The pulses, with a spectrum centered around $750 \mathrm{~nm}$ are directed into an ultrahigh vacuum chamber where they are focused onto a cold supersonic jet of randomly aligned acetylene molecules. The three-dimensional momenta of the resulting ionic fragments from a single molecule are recorded as described previously [9]. As at maximum only one molecule interacts with one laser pulse it becomes possible to measure the duration and the carrier-envelope (CE) phase of each of the few-cycle pulses delivered at the repetition rate of $5 \mathrm{kHz}$ on a single-shot basis [10-12] instead of actively stabilizing it, leading to improved accuracy. The peak intensity of the laser pulses on target was $1.5 \times 10^{14} \mathrm{~W} / \mathrm{cm}^{2}$, as determined from a separate calibration measurement using single ionization of argon atoms in circularly polarized light [13].

\footnotetext{
a e-mail: markus.kitzler@tuwien.ac.at
}

This is an Open Access article distributed under the terms of the Creative Commons Attribution License 2.0, which permits unrestricted use, distribution, and reproduction in any medium, provided the original work is properly cited. 

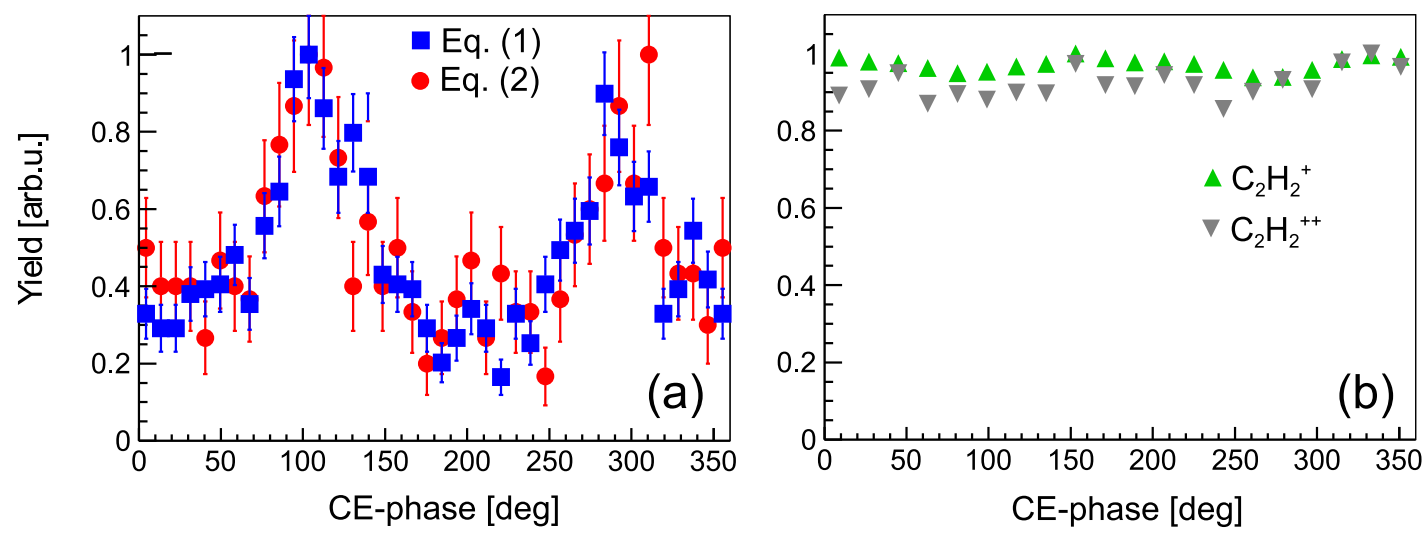

Fig. 1. Normalized ion yield as a function of CE-phase for fragmentation (a) and ionization (b) of acetylene. Squares and circles denote the fragmentation channels $\mathrm{C}_{2} \mathrm{H}_{2}^{2+} \longrightarrow \mathrm{CH}^{+}+\mathrm{CH}^{+}$and $\mathrm{C}_{2} \mathrm{H}_{2}^{2+} \longrightarrow \mathrm{C}_{2} \mathrm{H}^{+}+\mathrm{H}^{+}$, respectively, upward triangles and downward triangles denote the yields of the singly and doubly charged ions, respectively.

From our measured data we select all events where the molecule fragments via breakage of the center bond, Eq. (1), and via the ejection of a proton, Eq. (2), respectively:

$$
\begin{aligned}
& \mathrm{C}_{2} \mathrm{H}_{2}^{2+} \longrightarrow \mathrm{CH}^{+}+\mathrm{CH}^{+} \\
& \mathrm{C}_{2} \mathrm{H}_{2}^{2+} \longrightarrow \mathrm{C}_{2} \mathrm{H}^{+}+\mathrm{H}^{+} .
\end{aligned}
$$

In Fig. 1 we show that the normalized ion yields of these two fragmentation channels [Eqs. (1) and (2] exhibit an extraordinarily strong dependence on the $\mathrm{CE}$-phase, $\varphi_{\mathrm{CE}}$, with a modulation depth in excess of $70 \%$. The yields of the singly and doubly charged molecular ions, $\mathrm{C}_{2} \mathrm{H}_{2}{ }^{+}$and $\mathrm{C}_{2} \mathrm{H}_{2}^{2+}$, on the other hand show only a weak dependence on $\varphi_{\mathrm{CE}}$ (modulation depth $<10 \%$ ). The CE-phase was calibrated such that the mean momentum value of $\mathrm{C}_{2} \mathrm{H}_{2}^{+}$(see Fig. 2(b)) peaks at $\varphi_{\mathrm{CE}}=90^{\circ} / 270^{\circ}$ - as predicted by the simple man's model of strong field physics - although it is known that the maxima of the mean momentum value can be notably shifted due to the action of the ionic Coulomb potential [14] onto the departing electron. However, a possible $\varphi_{\mathrm{CE}}$ offset does not affect our explanations below.
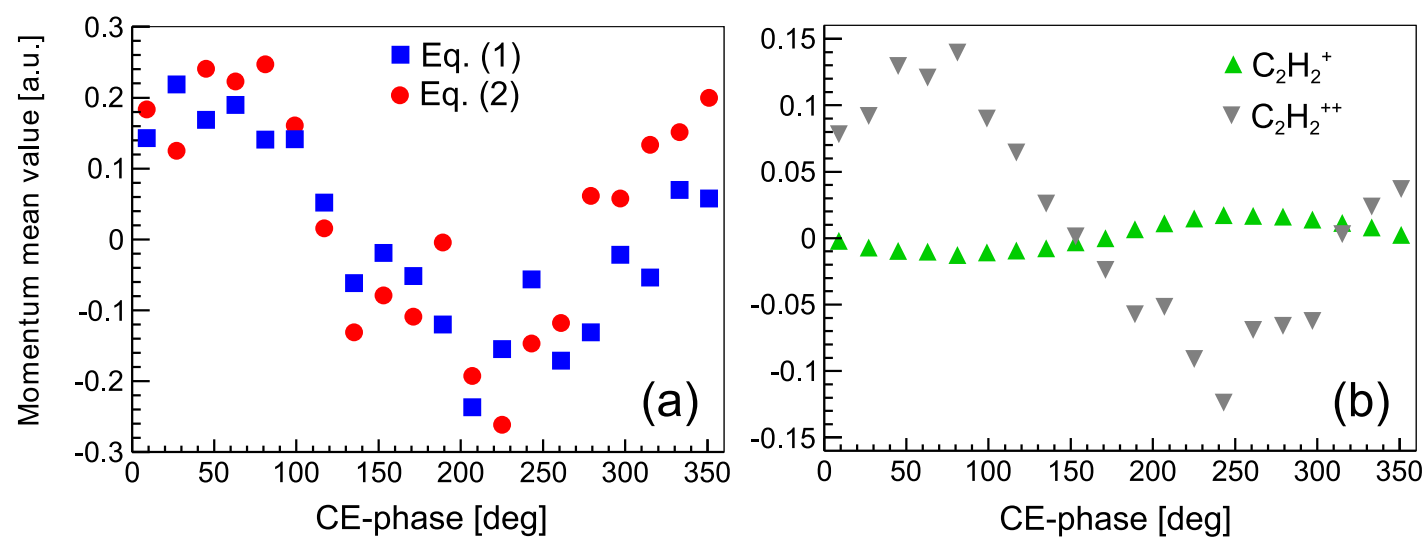

Fig. 2. Mean momentum values along the laser polarization direction for the two fragmentation channels (a) and the molecular ions (b). The same point styles as in Fig. 1 are used. For the fragmentation channels the sum momentum of the two ionic fragments is shown. 
In order to understand the mechanism by which the force associated with the sub-femtosecond light oscillations determines the relatively slow nuclear motion during the fragmentation reaction of this polyatomic molecule, we compare in Fig. 2 the mean sum momentum values along the laser polarization direction of the two fragmentation channels, Eqs. (1) and (2), as a function of $\varphi_{\mathrm{CE}}$, Fig. 2(a), with those of the molecular ions $\mathrm{C}_{2} \mathrm{H}_{2}{ }^{+}$and $\mathrm{C}_{2} \mathrm{H}_{2}^{2+}$ in Fig. 2(b). Due to momentum conservation the sum momentum equals the inverse of that of the two electrons detached during ionization. As can be seen, the sum momentum of the fragmentation channels shows maxima at almost the same $\varphi_{\mathrm{CE}}$ values as the mean momentum value of $\mathrm{C}_{2} \mathrm{H}_{2}^{2+}$. The mean momentum of $\mathrm{C}_{2} \mathrm{H}_{2}{ }^{+}$, however, shows maxima at different CE-phases - namely at the calibrated values $\varphi_{\mathrm{CE}}=90^{\circ} / 270^{\circ}$.

The intensity of $1.5 \times 10^{14} \mathrm{~W} / \mathrm{cm}^{2}$ used in our experiment is too small for sequential double ionization. Therefore, the doubly charged ion is expected to be dominantly created by impact ionization during electron recollision, i.e. via non-sequential double ionization. Electron recollision is a process that is driven by the laser electric field, and therefore highly sensitive to the CE-phase. The sum momentum that is imparted during the recollision ionization process to the two electrons is visible in the molecular ion(s). The fact that the mean momentum of the fragmentation channels Eqs. (1) and (2) peaks at the same CE-phases as the one of $\mathrm{C}_{2} \mathrm{H}_{2}^{2+}$ is strong evidence that the ionization step happens the same for both. Thus, the mechanism by which the fragmentation reactions Eqs. (1) and (2) are pre-determined by the laser light oscillations is electron recollision.

But how can electron recollision that takes place on sub-cycle times pre-determine a molecular fragmentation reaction that proceeds on a much longer time-scale? Especially the fragmentation reaction Eq. (1), whose time-scale is determined by the carbon motion, might be quite long. We propose the following scenario: Since the ground state of $\mathrm{C}_{2} \mathrm{H}_{2}^{2+}$ is a meta-stable state, excited states need to be reached before molecular fragmentation. During the recollision process, the second electron is kicked out from an inner-valence molecular orbital, which puts the dication into a dissociative electronically excited state from which it eventually fragments. The recollision energy in our experiment is right at the edge of imparting enough energy to the molecule for ionization from inner-valence orbitals. Only at certain CE-phases the recollision energy overcomes the ionization energy threshold. At these CEphases the fragmentation yield of a certain channel is strongly enhanced. As the recollision energy is high enough at all CE-phases to reach the ground state of the dication, the $\mathrm{C}_{2} \mathrm{H}_{2}^{2+}$ ionization yield is fairly independent of the CE-phase, see Fig. 1. Clearly, the control mechanism demonstrated here is strongly dependent on the laser intensity. This makes it a very universal method for laser driven molecular fragmentation control, as tuning the laser intensity allows adapting it to the energy level scheme of a specific molecule.

This work was partly financed by the Austrian Science Fund (FWF), grants P21463-N22 and V193-N16.

\section{References}

1. F. Remacle and R. D. Levine, PNAS 103, 6793 (2006).

2. J. Breidbach and L. Cederbaum, Phys. Rev. Lett. 94, 033901 (2005).

3. M. F. Kling et al., Science 312, 246 (2006).

4. I. Znakovskaya et al., Phys. Rev. Lett. 108, 063002 (2012).

5. I. Znakovskaya et al., Phys. Rev. Lett. 103, 103002 (2009).

6. Y. Liu et al., Phys. Rev. Lett. 106, 073004 (2011).

7. R. Weinkauf et al., J. Phys. Chem. 99, 11255 (1995).

8. M. Lezius et al., J. Chem. Phys. 117, 1575 (2002).

9. S. Roither et al., Phys. Rev. Lett. 106, 163001 (2011).

10. T. Wittmann et al., Nature Phys. 5, 357 (2009).

11. A. M. Sayler et al., Opt. Exp. 19, 4464 (2011).

12. A. M. Sayler et al., Opt. Lett. 36, 1 (2010).

13. C. Smeenk et al., Opt. Exp. 19, 9336 (2011).

14. S. Chelkowski, A. Bandrauk, and A. Apolonski, Phys. Rev. A 70, 013815 (2004). 